\title{
IUCD-related Knowledge, Attitudes and Practice among Primary Health Care Providers in Oman: Areas for Improvement
}

\author{
Asma AL Shidhani ${ }^{1 *}$, Fakhria Alrashdi², Aisha Albalushi ${ }^{2}$ and Maryam Al Shukri \\ ${ }^{1}$ Department of Family Medicine and Public Health, Sultan Qaboos University, Muscat, Oman \\ ${ }^{2}$ Primary Health Care, Ministry of Health, Muscat, Oman \\ ${ }^{3}$ Department of Obstetrics and Gynecology, Sultan Qaboos University, Muscat, Oman
}

\begin{abstract}
Objectives: The primary objective of this study is to assess three domains - the knowledge, attitude and practice related to the Intrauterine Contraceptive Device (IUCD) among primary health care providers (physicians and nurses) in Oman. The secondary objective is to compare the IUCD-related knowledge, attitude and practice of health care providers in primary health care between physicians and nurses.

Methods: A descriptive cross-sectional survey was conducted in primary health care centers in Muscat region of Oman from May, 2014 until September, 2015. The survey was a self-administered questionnaire contained questions to assess factual knowledge, attitudes and practice of IUCD. The questionnaire was distributed to female health care providers only. Data was collected by researchers and analyzed using SPSS version 20.

Results: Total 269 primary health care providers completed the questionnaire. $109(40.52 \%)$ participants had good and 131 (48.70\%) had average actual knowledge related to IUCD. The main reasons providers cited for not recommending the IUCD were concerns about the side effects; $174(64.68 \%)$ mentioned that it causes bleeding and $127(47.21 \%)$ reported that it needs service provider. Only $140(52.43 \%)$ of the providers would routinely recommend IUCD to their clients.

Conclusion: Future educational programs for health care providers should address the educational needs in the identified areas including misconceptions with regards to IUCD failure rate and side effects. Future training programs are also required to increase the technical competence of insertion and confidence in handling the side effects of IUCD.
\end{abstract}

Keywords: Family planning, nurse, doctors, IUCDs, Oman, contraception, health knowledge, attitudes, practice, surveys and questionnaires.

\section{INTRODUCTION}

Intrauterine device (IUCD) is a safe, long term reversible contraceptive method that can be used for most women with few side effects [1-3]. Worldwide this method is under-utilized despite being available and effective [1, 2, 4-6]. In Oman, since 1994 contraception services are provided to all married couples in primary health care centers including Cu38A IUCD [5, 7]. These services are provided by the government free of charge. Despite being available, accessible and free of charge, IUCD in Oman is underutilized [5, 8]. In 2008, National Reproductive Health Survey in Oman was conducted to evaluate family planning utilization and unmet needs, knowledge and attitude of IUCD use, maternal health care in married Omani females from 15 to 49 years of age. The survey revealed that the IUCD is less used compared to other contraceptive methods [5]. It also showed that health care providers are the main source of women's information about IUCD, and there is a deficit in the knowledge about IUCD benefits and side effects among Omani women [5, 9]. According to the yearly national health report released by Ministry of Health,

* Corresponding Author: Asma AL Shidhani, Department of Family Medicine and Public Health, Sultan Qaboos University, Muscat, Oman; Email:asmash@squ.edu.om

Received: December 30, 2019; Revised: February 27, 2020; Accepted: March 16, 2020

DOI: https://doi.org/10.37184/lnjpc.2707-3521.1.13
Oman, in 2012 the IUCD was the least utilized compared to other contraceptive methods. Clients' preferences were progesterone only pill (33.2\%), injectable $(28.4 \%)$, condom $18.1 \%$, Combined Oral Contraceptives (COC) $(11.7 \%)$ and IUCD (8.4\%). In the most recent report of 2017 , the IUCD had become the second least utilized with a marginal improvement to $13.6 \%$, followed by COC $(11.5 \%)[10,11]$. The majority of clients $(93.1 \%)$ were satisfied with the method they were using and intended to continue using it $[10,11]$. Across the years, more than $93 \%$ of clients have identified health care providers as the main source of their knowledge about contraceptive methods [10, 11].

Although utilization of contraception is influenced by many factors, there are evidence that lack of knowledge among clients and providers about IUCD is the main reason behind the under-utilization of IUCD [1, 7-9, 1214]. Studies have shown that physician knowledge, attitudes and practice do play a big role in the utilization of contraceptive methods in addition to the individual medical eligibility criteria [4, 15]. There are evidences in different populations that physicians are reluctant to recommend IUCDs except in narrowly selected patients due to several factors, including a lack of training in use of IUCDs during residency, a fear of litigation, and a belief that the IUCD creates a high risk for PID $[15,16]$. 
These concerns were not unique to primary health care providers but are also consistent for obstetriciangynecologists. When obstetricians and gynecologists were surveyed for their clinical use of IUCD, attitudes and how they select IUCD candidates; the results were similar to those of primary health care providers [17]. One important outcome of those studies that "many women could safely use the IUCD but are not offered" [17]. Educating physicians about the safety of IUCDs may expand their use and then more women would be offered this convenient, safe, and highly effective longterm contraception as shown by randomized clinical trials and expert opinion $[3,4,18,19]$. There are several studies exploring the end-user perspective in Oman on IUCD use [7-9, 11]. However, so far there are no published studies assessing IUCD-related knowledge, attitudes and practice among health care providers in Oman.

The primary objective of this study is to assess three domains -knowledge, attitudes and practice related to the IUCD as a contraceptive method among primary health care providers (physicians and nurses) in Oman. The secondary objective is to compare the IUCD-related knowledge, attitude and practice between physicians and nurses in primary health care.

\section{MATERIALS AND METHODS}

A descriptive cross-sectional survey was conducted in 2015 over a 5-month period (from May to September) in Muscat region, the capital area of Oman, assessing the knowledge, practice and attitudes of health care providers in primary health care facilities towards the use of Copper Intra-Uterine Device (IUCD) as a contraceptive method.

In 2015, Muscat region had total of 22 health centers. These health centers provide primary health care services including contraception services. Each health center had family physicians, general practitioners and nurses in addition to other technical and administrative staff. The nurses were involved in providing education about contraceptive methods and assisting doctors during the insertion procedure. The contraceptive services were provided only by female physicians and nurses. Twenty-one health centers of the total 22 were included in the study. One was excluded because it did not provide IUCD-insertion services. In those health centers, a total number of doctors and nurses were 525 (210 doctors and 315 nurses). As the total number of doctors and nurses working in Muscat health centers were 525 , the calculated sample size was 223 by taking level of confidence as $95 \%$ and margin of error as $5 \%$. Check market sample size calculator was used for sample size calculation [20]. Sample size was inflated up to $34 \%$ considering the non-response rate. Therefore, total 300 participants were approached.

Out of 300 forms distributed to 125 doctors and 175 nurses to make sure that both doctors and nurses were included in the study, 269 forms were filled with a response rate of $89.67 \%$. The researchers visited the health centers and supplied hard copies of the questionnaire to the head of the health center or to the chief staff nurse and the questionnaire was randomly distributed among the health center nurses and doctors. Doctors and nurses who agreed to participate were given a written consent form which was included in the questionnaire. The consent form contained information about the objective, nature of the research and the contact details of the researchers. Then the completed questionnaires were returned back to the researchers through the responsible person in each health center.

The self-administered questionnaire was modified from previous two studies investigating the IUCD-related knowledge, attitudes and practices among health care providers offering insertion of Copper IUCD and Levonorgestrel Intrauterine System (LNG-IUS) [15, 17]. The modifications were mainly made in the section assessing practice to fit the local practice in Oman. The study was approved by the Ministry of Health Research and Ethics Committee of the Directorate of Muscat. The survey had 4 parts with total 15 questions. The first was about the Socio-demographic characteristics of the participant and the second, third and fourth parts were on IUCD-related knowledge, attitude and practice respectively.

The knowledge was assessed through four multiplechoice questions. The first 2 questions requested the participant to indicate all the possible advantages and disadvantages of IUCD included in the list. The 2 questions together had 11 options which were all correct. Question one had 9 options and question two had 3 options. The third and fourth questions that were used to assess knowledge requested the participant to indicate the correct answer out of four possible options. One was regarding the failure rate of IUCD which is known to be less than $1 \%$, and the last question was regarding the duration of use of IUCD approved by American Food and Drug Administration (FDA) which is known to be 10 years.

The level of knowledge was measured by questions 1-4. According to the number of correct items indicated by the participants the level of knowledge will be scored. The total possible correct answers were 13. Level of knowledge was categorized as follows: poor knowledge if the participant scored 0 to 4 , average knowledge was 5 to 8 , and good knowledge was 9 to 13 .

The attitude was assessed by two questions. One was asking health care providers about the factors that might negatively influence their own decision to recommend IUCD by choosing from a list of potential or possible factors. Examples of those include; increases risk of PID, sexually transmitted infections (STI), irregular bleeding, dysmenorrhea, infertility, medical liability and associated expenses. They were also requested to indicate any 
other additional factors that have a role in their decision but not included in the list. The second question was asking health care providers to indicate why the clients might decline using the IUCD as a contraceptive method. They were offered a list of potential factors and were requested to add any other factors not included in the list.

The practice was assessed with four questions about attendance of IUCD insertion workshop for providers, attendance of educational activities like seminars, workshops or conferences around IUCD, and how many IUCD the provider has inserted in their carrier. In addition, health care providers were requested to indicate whether they do recommend IUCD for women who desire future pregnancy; routinely, only if other methods are unacceptable or unavailable or not at all. This question was included to assess the practice when it comes to this group of women who constituted the majority of women desiring contraception.

The data was analyzed using the Statistical Package for the Social Sciences (SPSS) version 20. Frequency and percentages were computed for categorical variables. Mean \pm standard deviation was used to express quantitative variables. Independent t-test was used to compare quantitative variable among two groups. Chisquare or Fisher-exact test was applied to compare qualitative variables among nurses and physicians. P-value of $<0.05$ was taken as statistically significant.

\section{RESULTS}

Of the 300 distributed questionnaires, with a response rate of $(89.67 \%), 269$ questionnaires were completed and returned from the participating health centers. The responders were $135(50.19 \%)$ primary health care physicians and $134(49.81 \%)$ nurses. The majority of participants $(n=196,72.86 \%)$ were 30 to 49 years of age, and $64(23.79 \%)$ were between $25-29$ years and few $(n=9,3.35 \%)$ were older than 50 years. Almost half $(n=141,52.41 \%)$ of the participants have been in practice for 5 to 14 years as shown in Table 1 .

Table 1: Socio-demographic characteristics of the participants.

\begin{tabular}{l|c|c}
\hline \multicolumn{1}{c|}{ Variables } & Groups & Frequency (\%) \\
\hline \multirow{4}{*}{ Age group } & $25-29$ years & $64(23.79)$ \\
\cline { 2 - 3 } & 30 to 49 years & $196(72.86)$ \\
\cline { 2 - 3 } & $\geq 50$ years & $9(3.35)$ \\
\cline { 2 - 3 } Marital status & Single & $33(12.27)$ \\
\cline { 2 - 3 } & Married & $235(87.36)$ \\
\hline Healthcare provider & Divorce & $1(0.37)$ \\
\cline { 2 - 3 } & Doctors & $135(50.19)$ \\
\hline \multirow{4}{*}{ Years of experience } & Nurses & $134(49.81)$ \\
\cline { 2 - 3 } & $\leq 4$ & $48(17.84)$ \\
\cline { 2 - 3 } & $5-14$ & $141(52.42)$ \\
\cline { 2 - 3 } & $15-24$ & $17(23.42)$ \\
\cline { 2 - 3 } & $\geq 25$ & $17.32)$ \\
\hline
\end{tabular}

In the knowledge domain, the questionnaire included a list of IUCD advantages. The majority $(n=227,84.39 \%)$ of health care providers agreed that IUCD provides long term protection while $186(69.14 \%)$ thought that it is very effective. The percentages for other advantages were also high for example; no interference with intercourse $(n=163,60.59 \%)$, no interference with breast feeding $(n=174,64.68 \%)$, no need for frequent follow up $(n=183$, $68.03 \%$ ) and immediate return of fertility once removed $(n=192,71.38 \%)$. When the knowledge of IUCD advantages was compared between doctors and nurses; in advantages list, the average items selected by doctors and nurses were 8 and 6.9 respectively that indicated the significant difference in the knowledge between the 2 groups. The doctors showed better knowledge than nurses $(p<0.001)$.

When the knowledge of disadvantages of IUCD was assessed, $106(39.41 \%)$ of the health care providers were aware that IUCD may cause prolonged and heavy period, $133(49.44 \%)$ knew that it may cause increased pain with periods or dysmenorrhea. $158(58.74 \%)$ of the health care providers agreed that it might cause vaginal discharge. When the knowledge of disadvantages of IUCD was compared between doctors and nurses, in disadvantages list the average items selected by doctors and nurses were 1.93 and 1.82 respectively and no significant difference was observed among doctors and nurses knowledge regarding disadvantages of IUCD $(p=0.335)$.

The third question on knowledge was about the failure rate for IUCD that is taken to be less than $1 \%$ per year. Of the physicians, $72(53.33 \%)$ correctly answered this question and $40(29.85 \%)$ of the nurses with $(p=0.002)$ which indicated a significant difference in the knowledge of this information between nurses and physicians. The last question to assess the knowledge was on the duration of use of Copper IUCD as approved by FDA which was 10 years. 94 (69.63\%) doctors have chosen the correct response while nurses were 70 (52.24\%) who correctly answered. Proportion of doctors who had correct knowledge regarding duration was significantly higher than nurses $(p=0.002)$.

When categorizing the health care providers according to level of IUCD-related knowledge, 29 (10.78\%) had poor knowledge, 131 (48.70\%) had average knowledge and 109 (40.52\%) had good knowledge. Table 2 compares the level of knowledge between physicians and nurses showing that physicians had more knowledge compared to nurses $(p<0.001)$.

In the Attitudes domain, in the providers opinion, the most frequent factors negatively influence patients' decision to use IUCD as a contraceptive method was the side effects of bleeding as indicated by $174(64.68 \%)$ of providers; $83(61.48 \%)$ of doctors and $91(67.91 \%)$ of nurses $(p=0.306)$. Followed by the fact that it needs a service provider for insertion as indicated by $79(58.52 \%)$ of the doctors and $48(35.82 \%)$ of the nurses. Health care providers were asked about factors that negatively 
Table 2: Comparison between doctors and nurses responses to the study questionnaire.

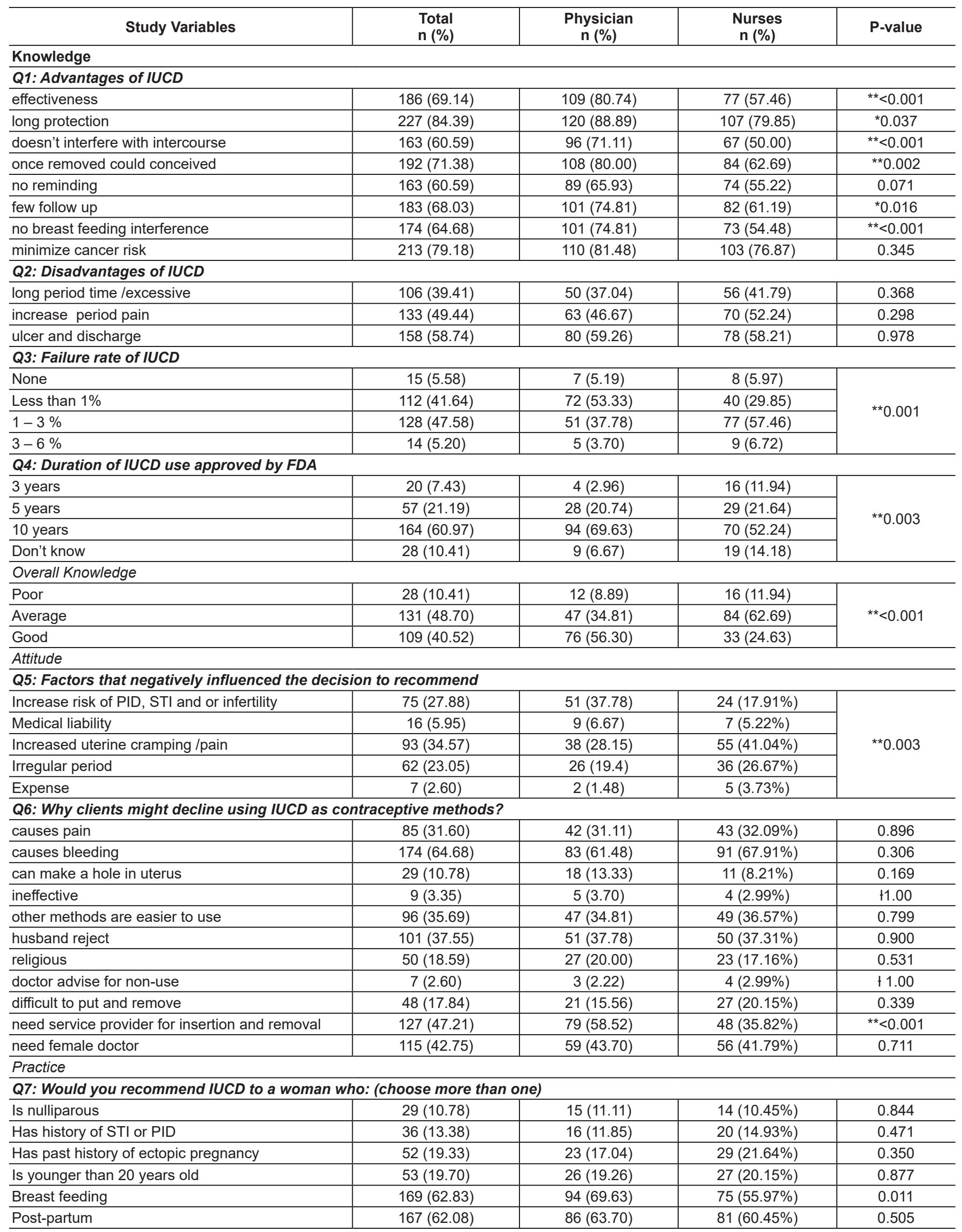




\begin{tabular}{|c|c|c|c|c|}
\hline \multicolumn{5}{|c|}{ Q8: Do you recommend the IUCD for women who desire more children in the future? } \\
\hline Recommend routinely & $140(52.43)$ & $86(64.44)$ & $54(40.30)$ & \multirow{3}{*}{$* *<0.001$} \\
\hline Recommend only if other methods are unacceptable & $97(36.32)$ & $36(27.06)$ & $61(45.52)$ & \\
\hline Never recommend & $30(11.15)$ & $11(8.15)$ & $19(14.18)$ & \\
\hline \multicolumn{5}{|l|}{ Q9: Any training program for IUCD? } \\
\hline Yes & $55(20.67)$ & $43(32.33)$ & $12(9.02)$ & \multirow{2}{*}{${ }^{* *}<0.001$} \\
\hline No & $213(79.18)$ & $91(67.41)$ & $122(91.04)$ & \\
\hline \multicolumn{5}{|l|}{ Q10: IUCD insertions techniques training? } \\
\hline Yes & $49(19.33)$ & $40(30.30)$ & $9(6.81)$ & \multirow{2}{*}{$* *<0.001$} \\
\hline No & $217(80.67)$ & $93(68.89)$ & $124(92.54)$ & \\
\hline \multicolumn{5}{|c|}{ Q11: How many numbers of IUCD inserted during your career? } \\
\hline None & $151(57.41)$ & $74(55.63)$ & $77(59.23)$ & \multirow{5}{*}{ *0.029 } \\
\hline $1-20$ & $63(23.95)$ & $26(19.54)$ & $37(28.46)$ & \\
\hline $21-50$ & $17(6.32)$ & $10(7.41)$ & $7(5.22)$ & \\
\hline $51-100$ & $14(5.20)$ & $8(5.93)$ & $6(4.48)$ & \\
\hline$>100$ & 18(6.69) & $15(11.11)$ & $3(2.24)$ & \\
\hline
\end{tabular}

f: Fisher-exact test is reported, * significant at $p<0.05,{ }^{* *}$ significant at $p<0.01$.

influenced their decision to recommend, the highest reported factor was increased uterine cramp/pain $(n=93$, $34.57 \%)$.

When assessing the IUCD-related practice; only 43 $(32.33 \%)$ of the doctors and $12(9.02 \%)$ of the nurses had attended an educational activity about IUCD, like a workshop or a conference. Of the nurses, $9(6.81 \%)$ had training on assisting in the insertion procedure. Of the physicians, $40(30.30 \%)$ had training on the IUCD insertion techniques. Of the physicians, $74(55.63 \%)$ never inserted IUCD in their carrier, 36 (27.07\%) inserted up to 50 IUCDs, and $15(11.11 \%)$ have inserted more than 100 during their carriers. Of the nurses, $77(59.53 \%)$ never assisted in IUCD insertion in their carrier, $44(33.84 \%)$ assisted in IUCD insertion up to 50 times, and $3(2.24 \%)$ have assisted in IUCD insertion more than 100 times during their carriers. More than half of healthcare providers $(n=140,52.43 \%)$ would routinely recommend IUCD to women desiring more kids in the future, $97(36.32 \%)$ do recommend it only if other methods are unacceptable and $30(11.15 \%)$ never recommend IUCD to a woman desiring future fertility. $86(64.44 \%)$ of doctors reported that they routinely recommend while nurses were only $54(40.30 \%)$ who responded that they routinely give recommendation for IUCD (see Table 2 for more details).

We attempted to establish an association between the level of knowledge of the health care provider and the likelihood of her practice to recommend IUCD for women desiring future pregnancy. $80(56.74 \%)$ of those with good knowledge would recommend IUCD and $7(23.33 \%)$ of those with poor knowledge would recommend it. Although it seemed that those with good knowledge were more likely to recommend IUCD compared to those with poor knowledge; the difference between the groups was not significant $(p=0.233)$.

\section{DISCUSSION}

On assessing the IUCD-related knowledge of primary health care providers in Oman, 109 (40.52\%) of them had good knowledge. This is relatively lower level of knowledge when compared to other studies where $69-78 \%$ of health care providers had good IUCDrelated knowledge $[15,17]$. In both of these studies the participants were all physicians. No previous studies assessed the level of IUCD-related knowledge among nurses involved in IUCD services. However, the level of knowledge varied depending on which aspect of knowledge was assessed. The knowledge related to the advantages of IUCD was good and $84.39 \%$ study participants were aware that IUCD provided long term contraception.

They were also aware that IUCD is effective, associated with quick return of fertility after removal and does not interfere with sexual intercourse and breast feeding. The poor knowledge was related to its failure rate, as only $41.64 \%$ of the health care providers $(53.33 \%$ physicians and $28.85 \%$ nurses) answered correctly. That was a disappointing finding as it is an important fact when counseling women for IUCD use. When comparing the level of knowledge between physicians and nurses, physicians had better knowledge than nurses in the collective IUCD-related knowledge, in the knowledge of IUCD-advantages, failure rate and FDA-approved duration of use. However, both professions were similar in knowing the disadvantages of IUCD.

Although insufficient knowledge among health care providers about IUCD is likely to discourage them from recommending it for use, in this study we were not able to establish a statistically significant association between the level of knowledge of the health care provider and the likelihood of recommending IUCD for contraception for women desiring future pregnancy. The reason was likely to be that generally there is a low preference of health care providers to recommend IUCD for use compared to other available methods like combined oral contraceptives [10, 11]. The other possible reason for the non-significant association between the knowledge and likelihood of recommending IUCD was that the knowledge measured here was a factual knowledge and 
did not necessarily mean a confidence in counseling, insertion and handling complications. This was the type of knowledge that was shown in several studies to really make a difference in practice $[3,4,19]$. The overall negative attitudes were illustrated by primary health care providers indicating several barriers that negatively influenced their decision to recommend IUCD as a contraceptive method. Those factors were mainly related to exaggerated concerns about the IUCD's safety and side effects which are the same factors reported in other studies $[15,17]$. Despite several studies confirming the safety of IUCD [1-3] the reported rate of PID related to IUCD-use was approximately 1.6 per 1000 womanyears of use [21]. However, in our study, still $27.88 \%$ healthcare providers put it as a concern for IUCD use. This finding of misconceptions and inadequate knowledge about IUCD is not unique to the health care providers in Oman [5, 9]. It has been shown in different health care settings including high income countries like United States of America (USA), low income countries like Nepal and specific ethnic groups like Navajo Nation which is a Native American group [15, 17, 18, 22, 23]. Primary health care providers in Oman also reported several reasons in their opinion why IUCD was underutilized by clients. Those factors were related to different aspects including the IUCD-side effects (heavy periods, dysmenorrhea and risk of uterine perforation), logistical (availability of female provider, ease of insertion, availability of other methods that were easier to use) and social factors like the opinion of the husband and religious concerns). These multiple factors from different aspects reflect the overall negative attitude regarding IUCD use among health care providers.

In a randomized clinical trial of health care providers conducted in USA, the whole team of health care providers in clinics providing contraception services were supplied with educational (didactic and hands-on) training for IUCD and compared with a control group in clinics that were not included in the training. Comparing the knowledge, attitudes and practices of the health care providers before the training and one year after the training showed significant improvement and also when comparing the health care providers between the trained group and the control group [3, 4, 19]. It is important for health policy makers in Oman to work on the issues of both clients and health care providers to optimize the utilization of the IUCDs, beyond the simple fact of making it available and accessible. We believe it is important to introduce changes to improve the utilization of the IUCD as a contraceptive method. Providing IUCD-training courses to all those involved in providing the service, monitoring the uptake and auditing practice are some of the ways we suggest to improve the utilization of IUCD as a contraceptive method.

Strengths of our study: It is the first in Oman to explore the role of health care providers' knowledge, attitudes and practice pertaining to IUCD utilization as a contraceptive method.

Since the study was limited to Muscat region as an urban area and no representation of more rural areas in the country this might limit the generalizability of the results to different socio-demographic settings. Also, it included primary health care centers from government sector with no representation from private health care sector. This is expected to have small if any effect on the quality of the data because the majority of contraception services are provided by government institutions.

\section{CONCLUSION}

Almost half of the primary health care providers have good IUCD-related knowledge and carry favorable attitudes towards IUCD use as a contraceptive method. Further training and mentoring should address the educational needs in the specific identified areas including misconception that IUCD increases risk of PID and to increase the technical competence of insertion and confidence in handling the side effects of IUCD. Such training opportunities are expected to improve the knowledge, liberate attitudes and boost the providers' confidence in the practice of counseling women for IUCD which in turn is expected to generate demand for this safe, reliable, long term and reversible contraceptive method.

\section{CONFLICT OF INTEREST}

We declare that there is no conflict of interest and no funding was received for this work.

\section{ACKNOWLEDGEMENTS}

We acknowledge heads of the health centers and the nurses in charge for assistance with data collection.

\section{REFERENCES}

1. Buhling K, Zite N, Lotke P, Black K. Worldwide use of intrauterine contraception: a review. Contraception 2014; 89(3): 162-73.

2. Black A, Guilbert E, Costescu D, Dunn S, Fisher W, Kives S, et al. Canadian contraception consensus (Part 3 of 4): chapter 7 - intrauterine contraception. J Obstet Gynaecol Can 2016; 38(2): 182-222.

3. Hov G, Skjeldestad F, Hilstad T. Use of IUD and subsequent fertility-follow-up after participation in a randomized clinical trial. Contraception 2007; 75(2): 88-92.

4. Thompson K, Rocca C, Stern L, Morfesis J, Goodman S, Steinauer $\mathrm{J}$, et al. Training contraceptive providers to offer intrauterine devices and implants in contraceptive care: a cluster randomized trial. Am J Obstet Gynecol 2018; 218(6): 597.e1-597.e7.

5. Al-Riyami A, AttiaAbd El Aty M, Jaju S, Morsi M, AlKharusi H, Al-Shekaili W. National Reproductive Health Survey 2008 (NRHS2008). Ministry of Health, Muscat, Sultanate of Oman.

6. Bahamondes L, Bottura B, Bahamondes M, Goncalves M, Correia V, Espejo-Arce X, et al. Estimated disability-adjusted life years averted by long-term provision of long acting contraceptive methods in a Brazilian clinic. Hum Reprod 2014; 29(10): 2163-70.

7. Al-Balushi MS, Islam MM, Ahmed MS, Khan MHR. Contraceptive method choices among women In Oman: a multilevel analysis. Data Sci J 2016; 14: 117-32. 
8. Al Kindi R, Al Sumri $H$. Prevalence and sociodemographic determinants of contraceptive use among women in Oman. East Mediterr Health J 2019; 25(7): 495-502.

9. Al-Balushi $M$, Ahmed $M$, Islam $M$, Khan $M$. Determinants of contraceptive use in Oman. Far East J Theor Stat 2015; 50(1): 5164.

10. Oman Ministry of Health. Annual Health Report 2012. Muscat; 2012.

11. Oman Ministry of Health. Annual Health Report 2017. Muscat; 2017.

12. Black A, Guilbert E, Costescu D, Dunn S, Fisher W, Kives S, et al. Canadian contraception consensus. J Obstet Gynaecol Can 2015; 37(10): 936-42.

13. Al Riyami A, Elaty MA, Morsi M, Al Kharusi H, Al Shukaily W, Jaju S. Oman world health survey: part 1 - methodology, sociodemographic profile and epidemiology of non-communicable diseases in Oman. Oman Med J 2012; 27: 425-43.

14. Van Zijl S, Morroni C, Van der Spuy Z. A survey to assess knowledge and acceptability of the intrauterine device in the Family Planning Services in Cape Town, South Africa. J Fam Plann Reprod Health Care 2010; 36(2): 73-8.

15. Callegari L, Darney B, Godfrey E, Sementi O, Dunsmoor-Su $\mathrm{R}$, Prager $\mathrm{S}$. Evidence-based selection of candidates for the levonorgestrel intrauterine device (IUD). J Am Board Fam Med 2014; 27(1): 26-33.
16. Hardeman J, Weiss BD. Intrauterine devices: an update. J Am Fam Physician 2014; 89(6): 445-50.

17. Espey E, Ogburn T, Espey D, Etsitty V. IUD-related knowledge, attitudes and practices Among Navajo area Indian Health Service Providers. Perspect Sex Reprod Health 2003; 35(4): 169-73.

18. Cleland J, Ali M, Benova L, Daniele M. The promotion of intrauterine contraception in low- and middle-income countries: a narrative review. Contraception 2017; 95(6): 519-28.

19. Harper C, Blum M, de Bocanegra H, Darney P, Speidel J, Policar $M$, et al. Challenges in translating evidence to practice. Obstet Gynecol 2008; 111(6): 1359-69.

20. How to determine population and survey sample size? Available at: https://www.checkmarket.com/blog/how-to-estimate-yourpopulation-and-survey-sample-size (Accessed on: January 10, 2014).

21. Bateson D, McNamee K, Briggs P. Newer non-oral hormonal contraception. BMJ 2013; 346: f341.

22. Daniele M, Cleland J, Benova L, Ali M. Provider and lay perspectives on i ntra-uterine contraception: a global review. Reprod Health 2017; 14(1): 119.

23. Chakraborty N, Murphy C, Paudel M, Sharma S. Knowledge and perceptions of the intrauterine device among family planning providers in Nepal: a cross-sectional analysis by cadre and sector. BMC Health Serv Res 2015; 15(1): 39. 